EVS27

Barcelona, Spain, November 17-20, 2013

\title{
Advanced VDC simulations of In-wheel electric vehicle using Carsim and Simulink
}

\author{
Hyunuk Ha${ }^{1}$, Jongmoo Kim ${ }^{1}$, Shiuk Chung ${ }^{1}$, Jangmyung Lee ${ }^{2}$ \\ ${ }^{1}$ Electric motor research center, KERI(Korea Electrotechnology Research Institute), sungjudong, sungsan-gu, \\ Changwon-si, Gyeongsangnam-do, Korea, Republic of, \\ hyunuk@keri.re.kr, kimjm@keri.re.kr, suchung@keri.re.kr \\ ${ }^{2}$ Dept. Of Electronic engineering, Pusan National University, jangjeon-dong, geumjung-gu, \\ Pusan, Korea, Republic of, \\ jmlee@pusan.ac.kr
}

\begin{abstract}
Conventional engine based vehicles inevitably have complicated structures due to lots of elements. Because of this characteristic, research, development, and also marketing are mainly conducted by the conglomerate, like GM, BMW, Honda, and Hyundai motors, etc. But environmental pollution and fuel exhaustion by the stated vehicle increase the necessity of EV(Electric Vehicle) as a representative of green car. First of all, the structure of EV is relatively simple, and energy transmission ratio of electric motor is more efficient than the engine based power train system. In addition to these, inwheel EV can estimate exact wheel torque, which is the most fundamental information for slip control, such as VDC(Vehicle dynamics control)/TCS(Traction control system)/ABS(Anti-lock brake system). Various kinds of expectable situations during EV's navigation have been simulated through the coordination between 'Carsim' and 'Simulink'.
\end{abstract}

Keywords: In-wheel EV, Direct torque control, VDC(Vehicle dynamics control), slip control

\section{Introduction}

Recently, most of car-manufacturers have concentrated on EV by the social needs to the green vehicles. First of all, EV can significantly decrease fuel consumption which causes serious environmental problems. In addition to this, EV is expected to realize demand oriented production system because of its relatively simple structure. Direct torque transmission structured EVs consist of in-wheel type and in-axis type. Both kinds of structures have their own pros and cons, such as inwheel EV could be controlled wheel by wheel, so this structure guarantees high stability, smooth cornering and ABS-like system through optimal torque distribution to each wheel. But it's vulnerable for external disturbances and impulsive shock which contains high frequency, and it requires precise speed control for straight driving. In this paper, various kinds of expectable situations during the EV's navigation have been simulated through the collaboration between 'Carsim' and 'Simulink'.

Carsim's fundamental features are highly focused at conventional power-train system, engine torque converter-transmission-differential gear-wheel. In 
order to simulate in-wheel $\mathrm{EV}$, make practical motor modeling in Simulink, and insert it into the in-wheel EV's power train instead of engine in the conventional power train system. Detailed simulation environment is shown at figure 2 in section 2. This paper consists of four sections, including introduction. Section 2 describe proposed VDC algorithm of in-wheel EV and important simulation environments, section 3 illustrates simulation results for most representative four cases, section 4 finally concludes and presents some future works.

\section{VDC algorithm of in-wheel EV}

\subsection{Conventional In-wheel EV researches}

In-wheel EV is mainly researched by the traditional motor manufacturer, especially Protean[1], Michelin[2], AISIN[3], NTN[4], etc.

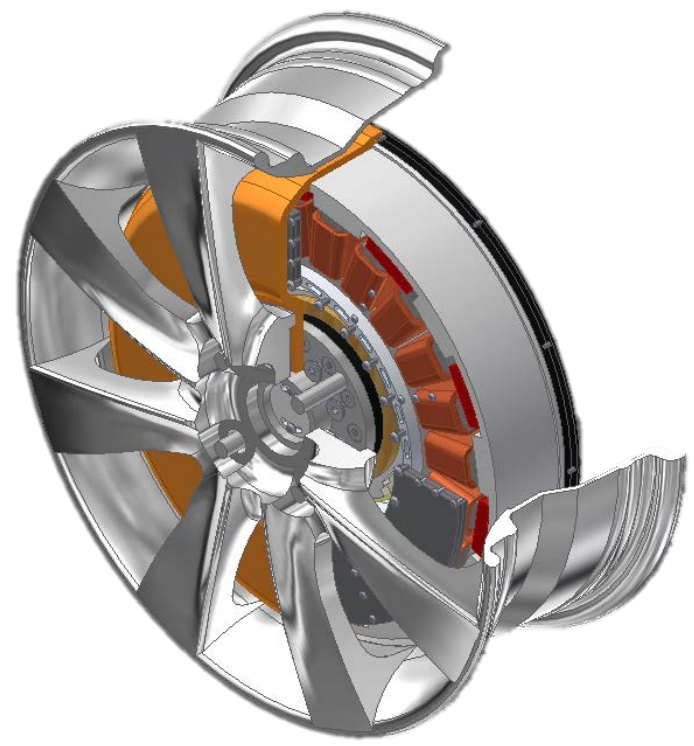

Figure 1 : Newly developed KERI's in-wheel motor

The most significant technologies for the inwheel $\mathrm{EV}$ is high torque density and robustness against external disturbances, and precise speed/torque control ability. Traditionally, the ratio between sprung mass and un-sprung mass affects vehicle's stability and driving comfort, so heavy weighted wheels diminish these two important vehicle characteristics. Robustness against external disturbances is directly related to maintenance problems and the life cycle of vehicle. Slip control of in-wheel EV is mainly conducted by Hori lab, the university of Tokyo[5]. Hori lab realized TCS by applying MFC(Model Following Control) in 2001[6], and recently proposed MTTE(Maximum transmissible torque estimation)[7] to enhance robustness as well as reliability.

\subsection{Proposed VDC algorithm for in- wheel EV}

Figure 2. illustrates overall block diagram of proposed VDC algorithm.

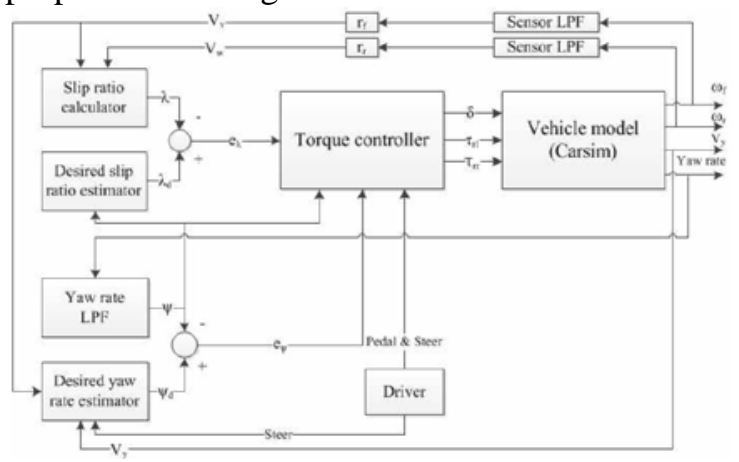

Figure 2 : Proposed VDC algorithm for in-wheel EV

Torque controller calculates wheel torque based on error of slip ratio, yaw rate, and driver' $\mathrm{s}$ accelerator pedal information. Vehicle model is replaced by Carsim block, slip ratio calculator attains forward wheel speed as estimator for vehicle speed, and rear wheel speed. The ratio between vehicle speed and wheel speed is defined as vehicle's slip ratio, and difference between desired slip ratio and estimated vehicle's slip ratio is controller input along vehicle's longitudinal axis. On the other hand, the between desired yaw rate and vehicle's yaw rate is another controller input along lateral axis. Detailed derivation of desired slip ratio and yaw rate is described in section 2.2.1 and 2.2.2, respectively.

\subsubsection{Desired slip ratio}

Fixed value, generally between $0.15 \sim 0.2$, is widely utilized in the former researches[8]. Figure 3. illustrates relationship between friction coefficient, mu, and vehicle's slip ratio, lambda by Pacejka's magic formula[9]. Most of the cases, except for cobblestone environment, road' $\mathrm{s}$ adhesion coefficient is maximized when vehicle's slip ratio is around 0.15 . So, conventional researches set desired slip ratio as fixed value, 0.15 . But vehicle's adhesion coefficient is varying by the vehicle's 
rotation elements. In this paper, desired varying slip ratio is determined as,

$$
\lambda_{d}=\lambda_{\text {default }}-0.05\left|\frac{\dot{\psi}}{\dot{\psi}_{\max }}\right|=0.15-0.05 \cdot\left|\frac{\dot{\psi}}{\dot{\psi}_{\max }}\right|
$$

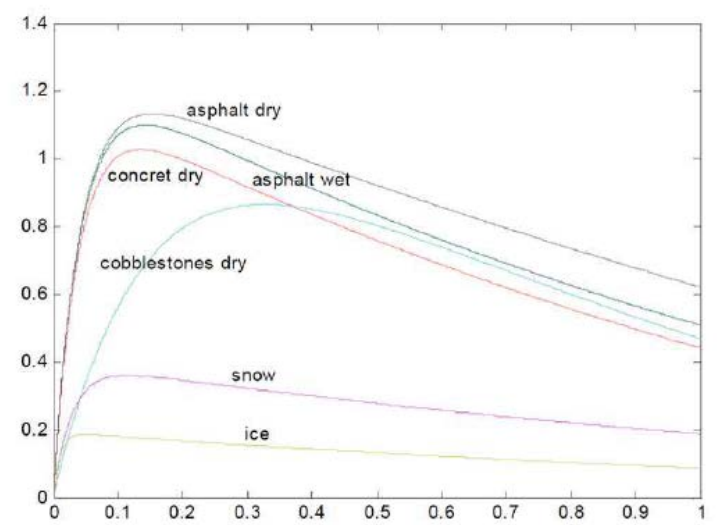

Figure 3 : Tire's mu-lambda curve

In default slip ratio is set as 0.15 as usual, and proposed desired slip ratio is varying according to the vehicle's normalized yaw rate as rotational information. Maximum yaw rate is tentatively selected as $100 \mathrm{rad} / \mathrm{s}$ by heuristic method, which is maximum yaw rate during slalom driving.

\subsubsection{Desired yaw rate}

By the vehicle's lateral dynamics in [10], yaw rate is stated as,

$$
\dot{\psi}=\frac{V_{x}\left\{\tan \left(\delta_{f}\right)-\tan \left(\delta_{r}\right)\right\} \cos (\beta)}{l_{f}+l_{r}}
$$

Where is $V_{x}$ is vehicle speed, $\delta_{f}$ and $\delta_{r}$ are front and rear wheel's steering angle, $\beta$ is vehicle slip, and finally $l_{f}$ and $l_{r}$ are distance between front wheel to COM and rear wheel to COM, respectively. Also from [10], vehicle's slip angle is derived as,

$$
\beta=\tan ^{-1}\left[\frac{l_{r} \tan \left(\delta_{f}\right)}{l_{r}+l_{f}}\right]
$$

In order to derive vehicle's desired yaw rate, (3) is substituted into (2). So we can attain vehicle desired yaw rate as in (4), which is a function of steering angle, vehicle's longitudinal and lateral speed when vehicle's COM is fixed.

$$
\dot{\psi}_{d}=\frac{V_{x} \tan \left(\delta_{f}\right) \cos \left(\tan ^{-1}\left(\frac{V_{y}}{V_{x}}\right)\right)}{l_{f}+l_{r}}
$$

\subsubsection{Wheel torque calculation algorithm}

In this paper, proposed wheel torque is calculated with the driver's accelerator pedal information, slip ratio error, and yaw rate error. Its detailed numerical formula is defined as,

$$
\begin{aligned}
\tau_{r l} & =T(P) \cdot\left\{1-(1-k) \frac{e_{\lambda}}{e_{\lambda \max }}\right\}\left\{1-k \frac{e_{\dot{\psi}}}{e_{\dot{\psi} \max }}\right\} \\
\tau_{r r} & =T(P) \cdot\left\{1-(1-k) \frac{e_{\lambda}}{e_{\lambda \max }}\right\}\left\{1+k \frac{e_{\dot{\psi}}}{e_{\dot{\psi} \max }}\right\}
\end{aligned}
$$

Where $\mathrm{T}(\mathrm{P})$ is driver's accelerator pedal, ${ }^{e_{\lambda}}$ is slip ratio error, $e_{\dot{\psi}}$ is yaw rate error, $\mathrm{k}$ is vehicle's rotational factor defined as,

$$
k=\left|\frac{\dot{\psi}}{\dot{\psi}_{\max }}\right|
$$

\section{Simulation}

\subsection{Simulation environment}

As mentioned in introduction, carsim 's default interface is established on engine based powertrain system. So we have to make a suitable motor model to simulate inwheel EV as fig. 4. shows carsim's internal setting so as tso in-wheel EV simulation and its fundamental simulation scheme. T-N curve(Rpmtorque curve) is designed using user defined function, which is torque command input of motor to Carsim. Other parameters, like tire radius/ friction constant/ overall length/ weight/ slanted angle of road is set in the Carsim as shown in table 1.

Table 1. Some important internal Carsim parameters

\begin{tabular}{lll}
\hline Category & Value & Unit \\
\hline Front wheel-COM & 1103 & $\mathrm{~mm}$ \\
Front wheel-Rear wheel & 2347 & $\mathrm{~mm}$ \\
Vehicle mass & 747 & $\mathrm{~kg}$ \\
Slanted angle & 0 & $\mathrm{deg}$ \\
Tire radius & 292 & $\mathrm{~mm}$ \\
Road friction coefficient & 0.85 & - \\
\hline
\end{tabular}




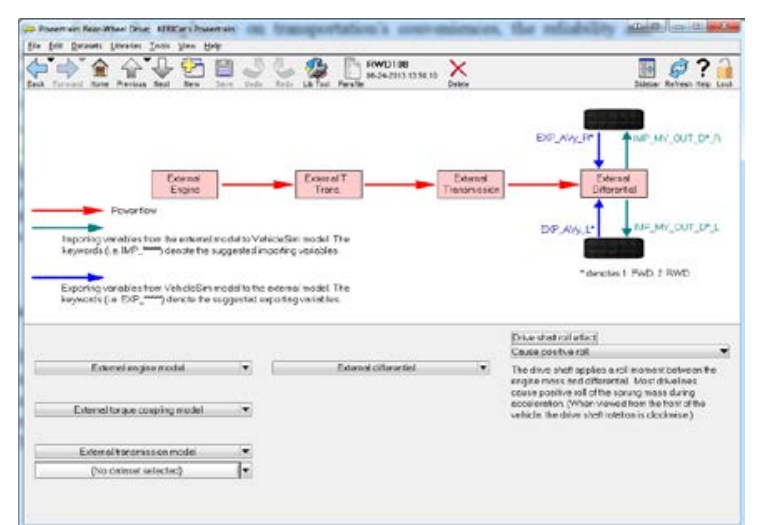

Figure 4 : Internal setting of Carsim for in-wheel EV and fundamental simulation scheme

We propose five most representative environments to simulate in-wheel $\mathrm{EV}$, which is split mu braking test(ISO14512), double lane change(ISO3888), slalom(S-turn), Circle, and J-turn. Our proposed VDC algorithm has been demonstrated under stated five environments by comparing slip ratio, lambda, slip angle, beta, tire's longitudinal and lateral force, and yaw rate, etc.

\subsection{Simulation result}

This section describes simulation results for stated environment. Each figure has its own short caption for most distinctive features.

\subsubsection{Double lane change(ISO3888)}
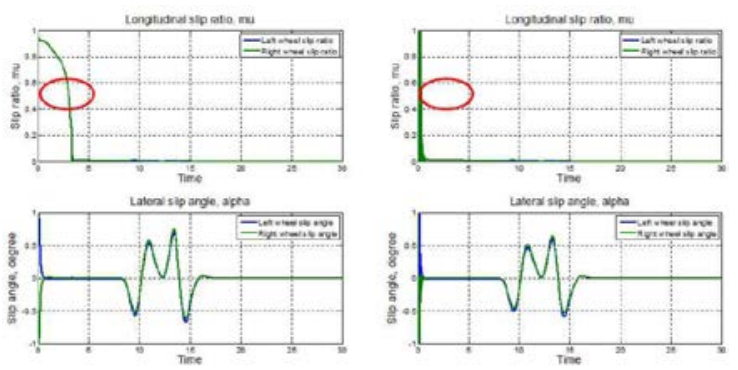

Figure 5 : Slip ratio and slip angle of without VDC/with VDC for double lane change
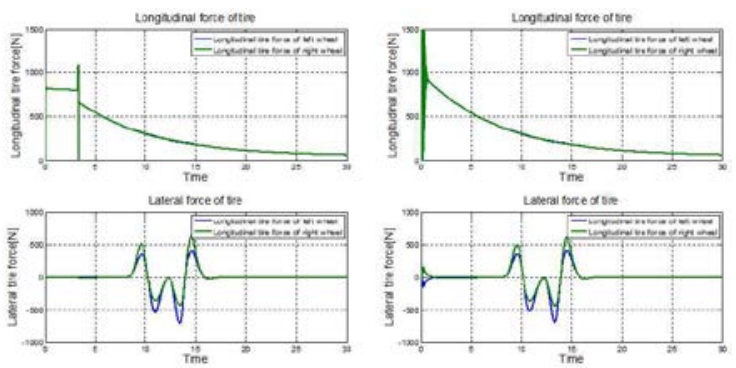

Figure 6 : Tire's longitudinal/lateral force of without VDC/with VDC

\subsubsection{Slalom(S-turn)}
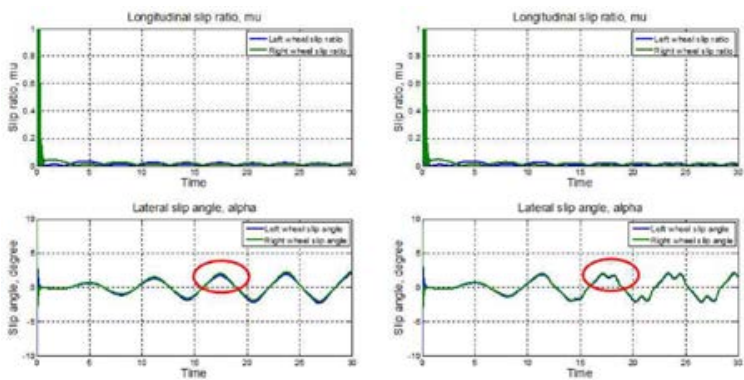

Figure 7 : Slip ratio and slip angle of without VDC/with VDC
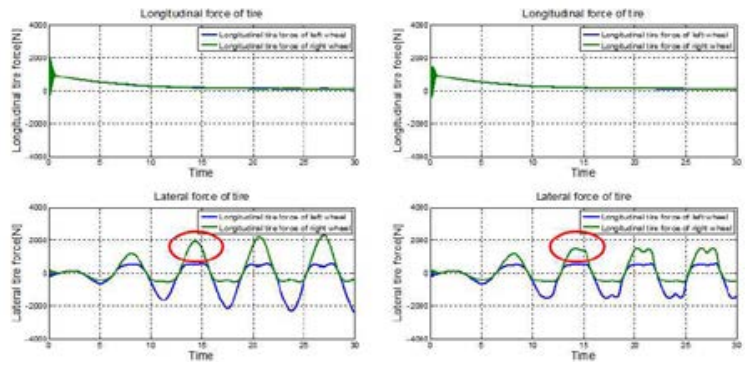

Figure 8 : Tire's longitudinal/lateral force of without VDC/with VDC
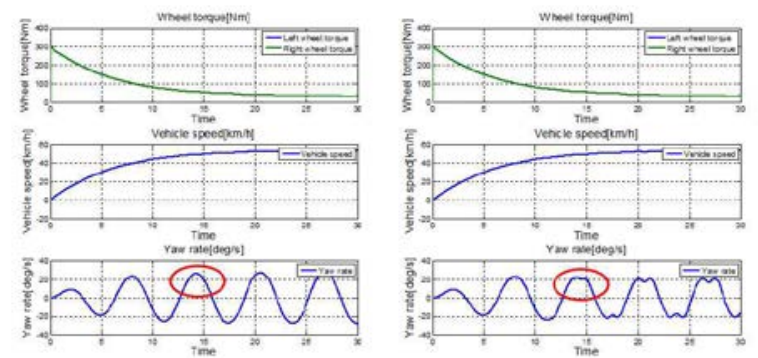

Figure 9 : Wheel torque, vehicle speed, and yaw rate for slalom simulation

\section{Conclusions}

Most EV experts have consentaneously announced that the most significant bottleneck of EV is battery problem. Its low energy density, short driving distance per full charging state, insufficient life cycle, and heavy weight are slowing EV down not to be next generation's transportation. But $\mathrm{EV}$ is an inevitable and huge wave heads for our future. As emphasize on transportation's conveniences, the reliability and stability issues should be considered concurrently. Moreover, in-wheel EV might be a good and the only solution for specialized EV fields, such as personal mobility system with distinctive chassis. This paper suggests in-wheel $\mathrm{EV}^{\prime} \mathrm{s}$ prospective forecasts, and stability problems. As a solution for the suggested issues, efficient VDC algorithm based on precise wheel torque/speed estimation has been proposed. Proposed algorithm has been demonstrated through some practical simulations with Carsim and Simulink. As some 
expected future works, 1 . Optimal torque distribution to minimize side-slip angle, 2. Substitute motor modeling to real motor to realize HILS(Hardware In the Loop Simulation) system.

\section{Acknowledgments}

This research was supported by the MOTIE(The Ministry of Trade, Industry and Energy), Korea, under the Human Resources Development Program for Special Environment Navigation/Localization National Robotics Research Center support program supervised by the NIPA(National IT Industry Promotion Agency)." (H1502-13-1001)

\section{References}

[1] http://www.proteanelectric.com/

[2] Espanet, C., Tekin, M.; Bernard, R.; Miraoui, A.; Kauffmann, J.-M.: A new structure of an high torque in-wheel motor,: Electrical Machines and Systems, 2003. ICEMS 2003. Sixth International Conference on, vol. 1, pp. 158-162, 2003.

[3] Ryoji Mizutani, Nishikamo-gun, Atsushi Torii, Shigetaka Isogai: In-wheel motor capable of efficiently cooling electric motor and reduction gear: United states patents, 1st ,Apr., 2008.

[4] Minoru Suzuki, Koichi Okada, Kayo Sakai, Yusuke Makino: Development of an InWheel Motor Axle Unit: NTN technical review, no, 75, 2007.

[5] http://hflab.k.u-tokyo.ac.jp/

[6] Dejun Yin, Yoichi Hori: A Novel Traction Control for Electric Vehicle without Chassis Velocity:

[7] Jia Sheng, Dejun Yin, Yoichi Hori, Feng rung hu: Electric vehicle traction control: IEEE trans. On Industry applications, vol. 18, no. 2, pp. 23-31. 2012.

[8] Rongrong Wang, Junmin Wang: Tire-road friction coefficient and tire cornering stiffness estimation based on longitudinal tire force difference generation: Control engineering practice, vol. 21 , no. 1 , pp. $65-$ 75. 2013.

[9] Mohammad Safwan Burhaumudin, Pakharuddin Mohd Samin, Hishamuddin Jamaluddin, Roslan Abd Rahman, Syabillah Sulaiman: Modeling and Validation of Magic Formula Tire Model: International Conference on Automotive, Mechanical and Materials Engineering (ICAMME'2012) Penang (Malaysia), May 19-20, 2012.

[10] Rajesh Rajamani: Vehicle Dynamics And
Control: Mechanical Engineering Series, ISSN 0941-5122, 3rd edition, 2011.

\section{Authors}

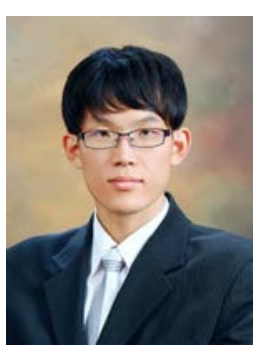

Hyunuk Ha received the B.Sc. and M.Sc degrees in Electrical Engineering from Pusan National University, Busan, Korea, in 2008, and 2010, respectively, where he is currently pursuing Ph.D degree. His current research interests are in-wheel EV and mobile inverted pendulum.

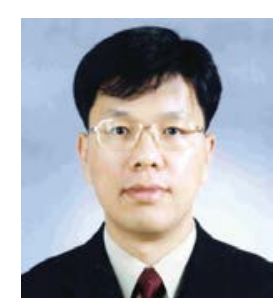

Jongmoo Kim born in Korea in 1970, He received the B.S., M.S., and Ph.D degrees in electrical engineering from Yeungnam University, Kyungbuk, Korea, in 1992, 1993, and 2009, respectively. Since 1994, he has worked with the Korea Electrotechnology Research Institute(KERI). His research interests are high performance motion and motor control, control theory, DSP application, contactless power supply and electric vehicle.

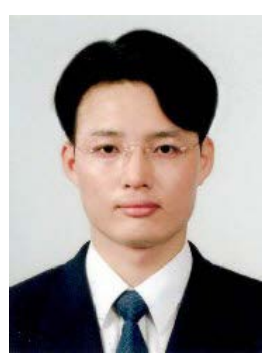

Shiuk Chung received the B.S, M.S. and Ph.D degrees in mechanical engineering from Pusan National University, Busan, S.Korea in 1997, 1999, and 2010, respectively. From 2002 to 2005, he was with Samick THK as a researcher. Since 2005, He has been with Electric Motor Research Center, Korea Electrotechnology Research Institute, Changwon, S.Korea, as a Seniotr Researcher. His research interests include the design and analysis of linear and rotary direct drive electric machines.

Jangmyung Lee received the B.S and M.S degrees in electronic engineering from Seoul National University, Seoul, Korea, in 1980 and 1982, respectively, and the $\mathrm{Ph}$. D degree in computer engineering from the University of Southern California (USC), Los Angeles, in 1990. Since 1992, he has been a Professor at Pusan National University, Busan, Korea. His research interests include intelligent robotics, advanced control algorithm, and specialized environment navigation/localization. 